\title{
EARLY EFFECTS OF CARBACHOL ON THE MORPHOLOGY OF MOTOR ENDPLATES OF MAMMALIAN SKELETAL MUSCLE FIBERS
}

TILMAN VOIGT, PhD

Institute of Anatomy, University of Fribourg; Institute of Anatomy, University of Bern, Switzerland

ABSTRACT: Long-term disturbance of the calcium homeostasis of motor endplates (MEPs) causes necrosis of muscle fibers. The onset of morphological changes in response to this disturbance, particularly in relation to the fiber type, is presently unknown. Omohyoid muscles of mice were incubated for 1-30 minutes in $0.1 \mathrm{mM}$ carbachol, an acetylcholine agonist that causes an inward calcium current. In these muscles, the structural changes of the sarcomeres and the MEP sarcoplasm were evaluated at the light- and electron-microscopic level. Predominantly in type I fibers, carbachol incubation resulted in strong contractures of the sarcomeres underlying the MEPs. Owing to these contractures, the usual beret-like form of the MEP-associated sarcoplasm was deformed into a mushroom-like body. Consequently, the squeezed MEPs partially overlapped the adjacent muscle fiber segments. There are no signs of contractures below the MEPs if muscles were incubated in carbachol in calcium-free Tyrode's solution. Carbachol induced inward calcium current and produced fiber-type-specific contractures. This finding points to differences in the handling of calcium in MEPs. Possible mechanisms for these fiber-type-specific differences caused by carbachol-induced calcium entry are assessed.

Carbachol is an acetylcholine agonist. When applied chronically, it causes a profound destruction of the myofibrils underlying the motor endplate (MEP). This effect is attributable to dissolution of the Z-line. ${ }^{1,2}$ The pathological changes are reputed to be calcium-dependent ${ }^{1,2}$ and are comparable with those manifested in patients with slow-channel syndrome. ${ }^{3}$ Similar calcium-dependent destruction of the myofibrils beneath the MEP can be elicited by daily application of an acetylcholinesterase inhibitor. ${ }^{4,5}$ The effect is brought on by prolonged opening of the acetylcholine receptors (AChRs) and ensuing entry of calcium in the area of the MEP. A genetic manipulation of the AChRs that prolongs the opening time of the receptor channels causes a slow-channel-syndrome-like pathology in mice. ${ }^{6}$ However, not all skeletal muscle fibers are affected. ${ }^{6}$ This selectivity accords with observations relating to the effects of carbachol. ${ }^{1,2}$ According to earlier data, carbachol affects preferentially type I fibers. This, combined with the observation that type I fibers are predominantly damaged by ACh esterase inhibitors, ${ }^{4}$ suggests a greater vulnerability to calcium homeostasis in these fibers.

Abbreviations: AChR, acetylcholine receptor; ANOVA, analysis of variance; MEP, motor endplate

Key words: calcium; carbachol; fiber type; morphology; motor endplate Correspondence to: T. Voigt; e-mail: voigt@ana.unibe.ch
Little is known of the early morphological changes associated with short-term carbachol stimulation. To improve our understanding of the disturbances in MEP calcium homeostasis that are elicited by the entry of calcium through activated AChRs, it would be beneficial to temporally and topographically pinpoint the first visible signs of damage and to ascertain whether the changes are fiber-specific. To address these questions, the omohyoid muscle of mice was stimulated with carbachol under isometric conditions for 1-30 minutes. The treated muscles were evaluated at the light- and transmission-electron-microscopic level. Morphological changes in the MEP, as well as the grades of contraction and the types of fibers, were assessed.

\section{METHODS}

Following anesthesia of the mice [NMRI (Han) outbred, 3-5 months old, 3 animals per experimental set-up] with a mixture of xylazine (Bayer), and ketamine (Parke, Davis \& Co.) the hearts of the mice were excised and the skin over the neck was opened. After removing the cervical glands, the sternocleidomastoid, cleidomastoid, and clavotrapezoid muscles were resected, beginning at the cranial end. ${ }^{7}$ The lateral margin of the omohyoid muscle-between the posterior belly of the digastric muscle and the clavicles-was then exposed. The major and minor pectoral muscles were cut away from their origins, and the clavicles were disconnected from the sternum and the scapula. The subclavian artery and vein, together with the surrounding connective tissue, were removed. The subscapular muscle was exposed and reduced to reveal the attachment of the omohyoid to the scapula. The omohyoid muscle was then partially, but not completely, detached from the scapula so that it remained connected to a piece of bone. The sternohyoid and the sternothyroid muscles were cut away from the sternum, and the omohyoid muscle was detached from the reverse side of the former. The posterior venter of the digastric muscle was resected, and the muscular connection between the hyoid and the tongue was severed. The hyoid bone was cut with a pair of scissors, and the thyrohyoid muscle at the back of the omohyoid was detached.

At a resting length of about $2.5 \mathrm{~cm}$, the muscles were pinned to cork plates, which were 


\begin{tabular}{|c|c|c|c|c|c|}
\hline$C(n=3)$ & $E_{1}(n=3)$ & $E_{2}(n=3)$ & $E_{3}(n=3)$ & $\mathrm{R}(n=3)$ & wCa $(n=3)$ \\
\hline $\begin{array}{l}30 \text { min in } \\
\text { Tyrode's solution }\end{array}$ & $\begin{array}{l}1 \text { min carbachol in } \\
\text { Tyrode's solution }\end{array}$ & $\begin{array}{l}10 \text { min carbachol } \\
\text { in Tyrode's solution }\end{array}$ & $\begin{array}{l}30 \text { min carbachol in } \\
\text { Tyrode's solution }\end{array}$ & $\begin{array}{l}30 \text { min carbachol in } \\
\text { Tyrode's solution } \\
\text { 150-min rinse in } \\
\text { Tyrode's solution }\end{array}$ & $\begin{array}{c}30 \text { min carbachol in } \\
\text { Tyrode's solution } \\
\text { without } \mathrm{CaCl}_{2}\end{array}$ \\
\hline Fixation & Fixation & Fixation & Fixation & Fixation & Fixation \\
\hline
\end{tabular}

immersed in Tyrode's solution [ $\mathrm{NaCl} 137 \mathrm{mM}, \mathrm{KCl}$ $5 \mathrm{mM}, \mathrm{MgCl}_{2} 1 \mathrm{mM}, \mathrm{CaCl}_{2} 2 \mathrm{mM}$, HEPES $10 \mathrm{mM}$, glucose $10 \mathrm{mM}(\mathrm{pH} 7.4)]$ containing $0.1 \mathrm{mM}$ carbachol (Sigma). They were incubated therein for 1-30 minutes at $20^{\circ} \mathrm{C}$ (Table 1). As controls, muscles were incubated in Tyrode's solution. To address the question of whether the effects of carbachol incubation are calcium-dependent, muscles were incubated in $0.1 \mathrm{mM}$ carbachol in Tyrode's solution without calcium. In another group, the carbachol was rinsed off with Tyrode's solution after 30 minutes of carbachol incubation. The muscles were fixed by immersion in $2.5 \%$ glutaraldehyde in $0.1 \mathrm{M}$ cacodylate buffer ( $\mathrm{pH} 7.4$ ) for $1 \mathrm{~h}$ at $20^{\circ} \mathrm{C}$. The regions containing the MEPs were then excised, and the resulting blocks of tissue were trimmed. The blocks were postfixed in 1\% osmium in $0.1 \mathrm{M}$ cacodylate buffer, dehydrated in ethanol, and embedded in Epon.

From each muscle, a series of 140 semithin sections ( $1 \mu \mathrm{m}$ thick) were prepared with a histo-diamond (Diatomae) and stained with Richardson stain. $^{8}$ Each series of 140 semithin sections contained about 90-120 muscle fibers.

According to their aspect in the region of the fiber nuclei, the fibers were classified as either type I or II, using previously established criteria., 10 Type I fibers (or C-fibers) were characterized by an abundance of subsarcolemmal mitochondria, numerous strands of longitudinally oriented interfibrillar mitochondria, small irregular myofibrils, and the presence of many lipid droplets. Type II fibers (or A- and B-fibers) were characterized by few or no subsarcolemmal mitochondria, by few or no strands of longitudinally oriented interfibrillar mitochondria, and by much larger myofibrils.

From each series of 140 sections (per muscle and animal), 5 MEPs of type I fibers and 15 of type II were selected randomly. The fiber region around the MEPs as well as the region surrounding the next muscle fiber nucleus of the same fiber were photographed at a final magnification of $\times 1000$. The number of sarcomeres in the image of the muscle fiber and the number in the image of the associated MEP were counted. The ratios of these numbers were represented as the mean for the experimental set-up in question (Fig. 1). In addition, the quotient between the result of type I and type II fibers per animal was calculated and represented the mean for the experimental set-up in question (Fig. 1). Significance levels, as determined by analysis of variance (ANOVA), ${ }^{11}$ are shown in Table 2.

Ultrathin sections (70-100 nm in thickness) were prepared using a diamond (Diatomae), and then stained with uranylacetate and lead citrate. ${ }^{12}$ The sections were examined using a transmission electron microscope (Philips EM100), which was equipped with a digital camera (11-megapixel sidemounted TEM camera; Morada).

\section{RESULTS}

The incubation of muscles at resting length, in either Tyrode's solution alone (protocol C) or carbachol-containing Tyrode's solution without $2 \mathrm{mM}$ $\mathrm{CaCl}_{2}$ (protocol wCa) for up to 30 minutes at $20^{\circ} \mathrm{C}$, elicited neither histological (light-microscopic) nor ultrastructural (electron-microscopic) changes in either the muscle fibers or the MEPs (data not shown). Beneath the MEP, the pattern of sarcomeric striation was the same as that in the rest of the muscle fiber. Consequently, the ratio of numbers of sarcomeres in the image of the muscle

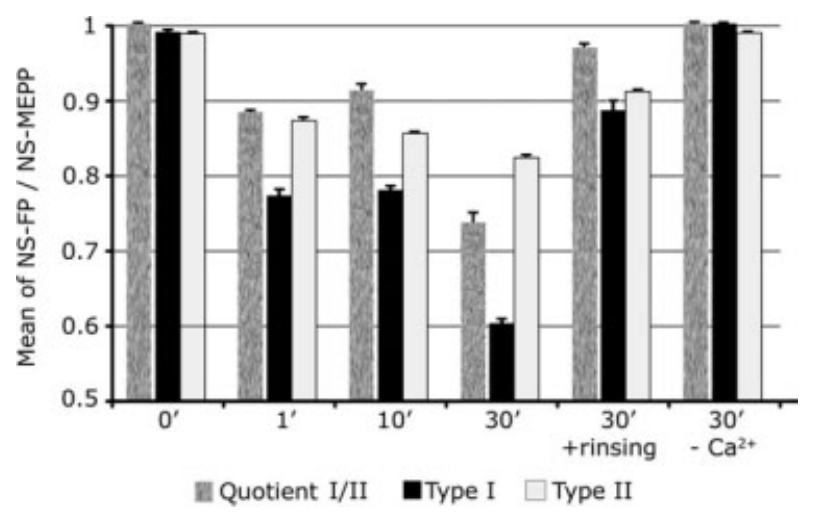

FIGURE 1. Mean and SEM data for the ratio of number of sarcomeres per muscle fiber photo (NS-FP) to number of sarcomeres per MEP photo (NS-MEPP). Each bar triplet represents the mean of three animals per time-point. Data for type I fibers represent the mean of 5 randomly selected type I fibers from each animal. Data for type II fibers represent the mean of 15 randomly selected type II fibers from each animal. 
Table 2. Significant differences between sarcomere number ratios of experimental groups according to analysis of variance. ${ }^{11}$

\begin{tabular}{|c|c|c|c|c|c|c|}
\hline & $C$ vs. $E_{1}$ & C vs. $E_{2}$ & C vs. $E_{3}$ & C vs. R & $E_{3}$ vs. R & $E_{3}$ vs. wCa \\
\hline Type I & + & + & + & - & + & + \\
\hline Type ॥ & - & + & + & - & - & + \\
\hline Quotient I/II & - & - & + & - & + & + \\
\hline
\end{tabular}

Statistical significant differences $(P<0.05)$ are indicated by + .

fiber to the number of sarcomeres in the image of the associated MEP was about 1:1 for fiber types I and II (Fig. 1).

In a few instances, treatment of muscles with carbachol led to local disturbances in the pattern of sarcomeric striation. These sarcomeric disarrangements were observed in all fiber types and were scattered in regions that were devoid of
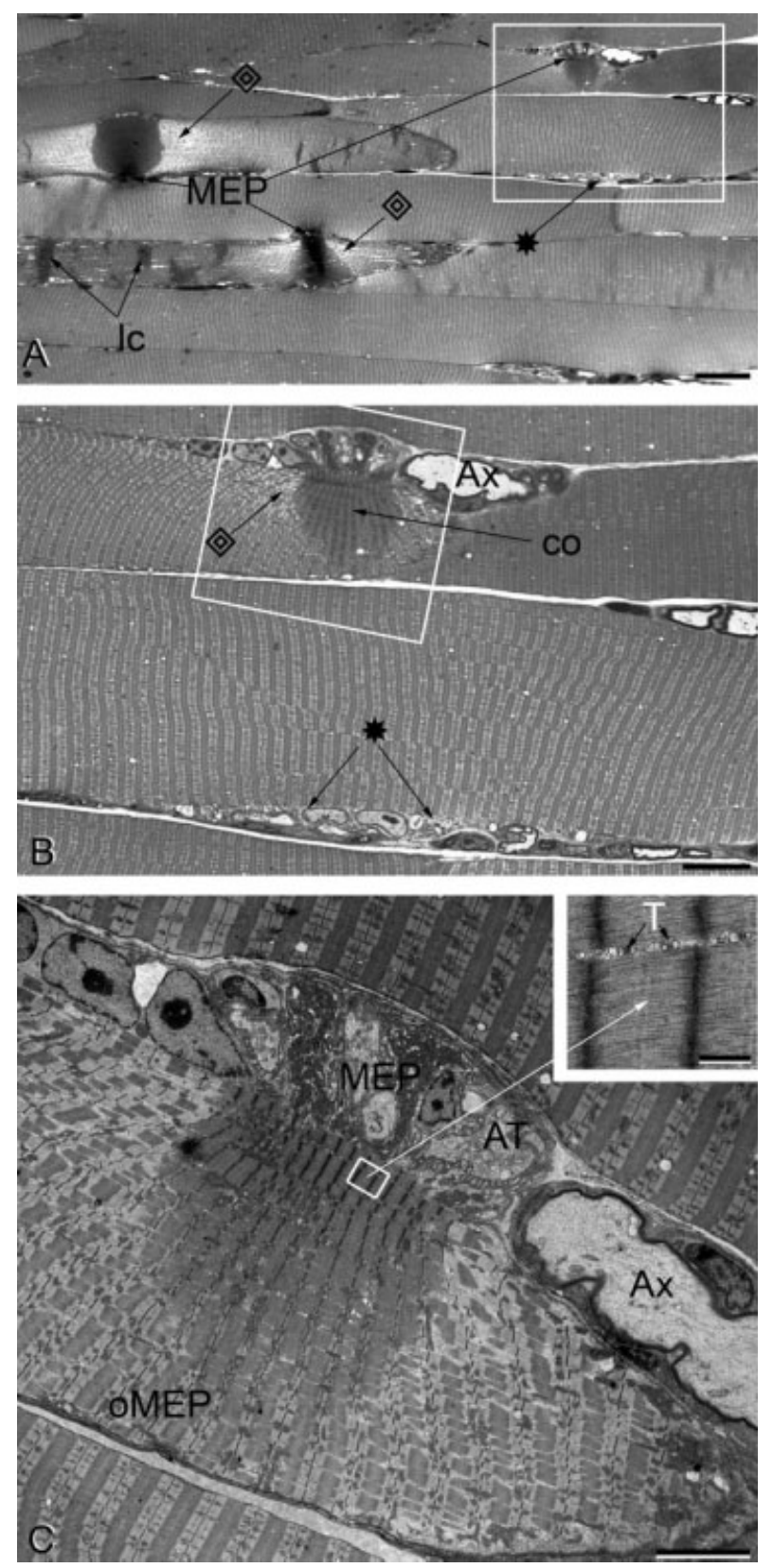

MEPs. They ranged from misalignments in register to total loss of striation (Fig. 2A). However, most fibers showed no sign of disturbance of striations after 1-30-minute treatment with carbachol, except for the MEP region (Figs. 2-4). In this region, beneath the MEPs, treatment with carbachol caused more or less local shortenings of the sarcomeres, leading to a magnetic-field-line-like image of the striation, with its epicenter under the MEP (Figs. 2 and 3C). The most pronounced shortenings were observed within the group of type I fibers (Figs. 2B and 3B), whereas the less-pronounced shortenings were found within the group of type II fibers (Figs. 2B and $3 \mathrm{~B}$ and $\mathrm{C}$ ).

In some instances, the sarcomeres were so condensed as to appear as a dense band (Fig. 3A and D) or spot (Fig. 3B). This type of contracture was unique to MEPs and could be readily distinguished from the milder, scattered disturbances of striation that were observed at other locations in the muscle fiber (Fig. 2A). If the local contracture was severe and if the muscle fibers were not densely packed, the MEPs appeared to be squeezed out of the muscle fiber, with the result that their usual beret-like form was transformed into a wart- or mushroomshaped form. This body, which contains the soleplate nuclei, sat upon and overlapped the muscle fiber (Figs. 3A, B, and D, and 4A). Owing to the plane of sectioning, some MEPs revealed two

FIGURE 2. Longitudinal section through the region of motor endplates of a skeletal muscle after incubation in $0.1 \mathrm{mM}$ carbachol for $10 \mathrm{~min}$ (protocol $\mathrm{E}_{2}$ ). (A) Local contractures are associated with several motor endplates (MEP), but beneath the others no deformation of the sarcomeric striation can be seen (*). Sarcomeres bordering contracted motor endplates in the up- and downstream directions are stretched $(\hat{\Delta})$. Some of the fibers manifest local contractures (Ic) (semithin section; bar $=25 \mu \mathrm{m}$ ). (B) Higher magnification view of the boxed area in (A) illustrating, in greater detail, a motor endplate with a local contracture (co) and one without (*), as well as the stretching of neighboring sarcomeres (仓). Ax, axon (bar $=10 \mu \mathrm{m}$ ). (C) Detail of the boxed area in (B) illustrating the contracture beneath the beretlike profile of the motor endplate (MEP). Within the contracture, the sarcomeric striations are condensed. On the opposite side of the motor endplates, the striation pattern is undisturbed (oMEP). Ax, axon; AT, axon terminal (ultrathin section; bar $=5 \mu \mathrm{m}$ ). (C) Inset: Higher magnification view of the boxed region in (C) illustrating, in greater detail, the condensation of the banding pattern in the contracted region. The M-line is visible but the I-band is not. The distance between neighboring triads $(T)$ of the sarcomere is greatly reduced (bar $=500 \mathrm{~nm}$ ). 
intersections of the same axon terminal, with two well-separated contractures (Fig. 3B). In cross-sectioned MEPs, the contractures were apparent as band-like regions within the underlying myofibrils, which were no longer organized into the usual hexagonal units that usually represent the A- and I-bands and the Z-line, respectively (Fig. 4).

Depending on the severity of contracture as well as the transverse and longitudinal distance to the MEP sarcoplasm, the normal periodicity of the sarcomeric striation was lost (Fig. 2C). Therefore,
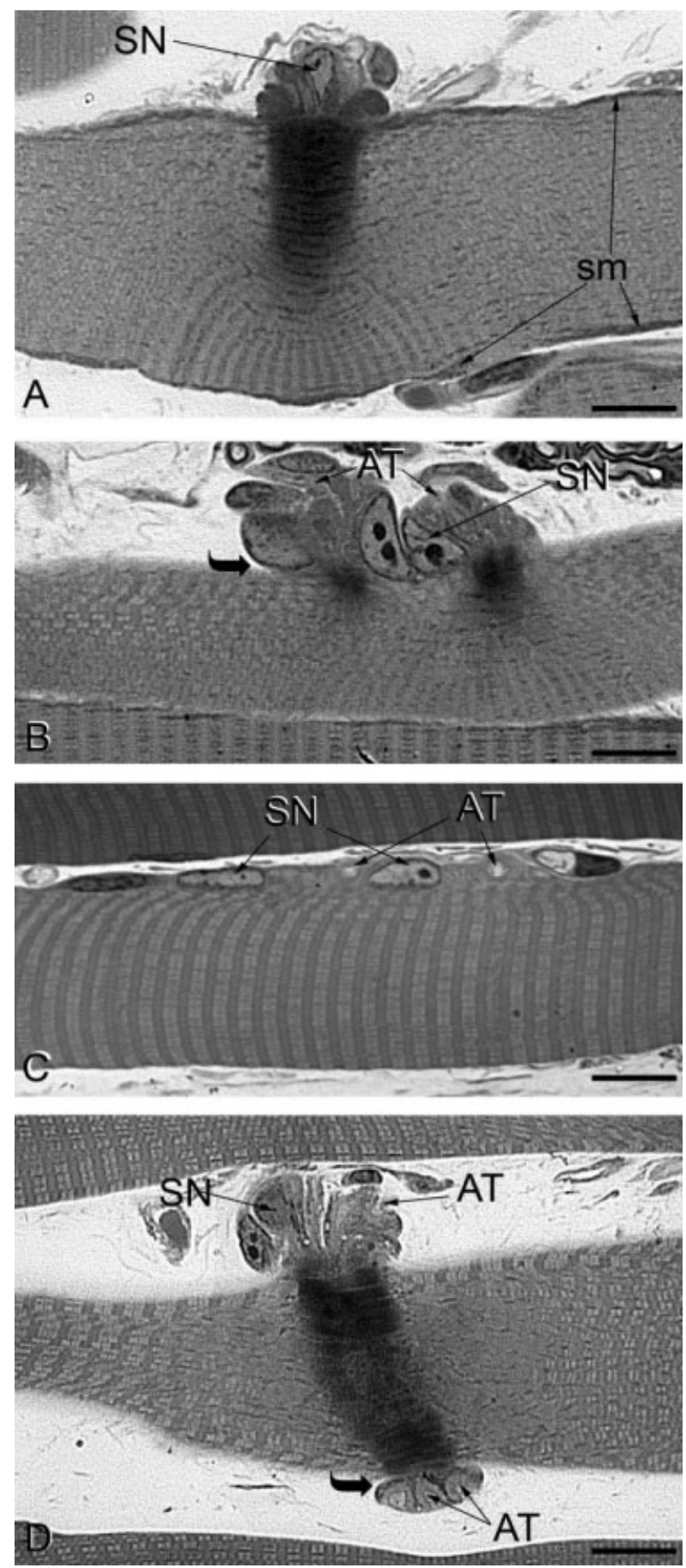

the M-lines disappeared even in cases of a spot- to band-like contracture (not shown). If the contracture was located deeper within the body of the muscle fiber and was more widespread in the transverse and longitudinal directions, then the M-line was just discernible (Fig. 2C) and, in most instances, the subsarcolemmal sarcomeres on the side opposing the MEP had a normal appearance (Fig. 2C). The distance between adjacent Z-lines (sarcomere length) ranged from $320 \mathrm{~nm}$ (in instances of severe contracture immediately beneath the MEP) to $2.4 \mu \mathrm{m}$ in the subsarcolemmal space opposing the affected MEP. Sarcomeres that border contracted sarcomeres were often in a stretched condition (Fig. 2).

The fiber-type-specific ratio of the number of sarcomeres per muscle fiber photo to sarcomeres per MEP image declined with increasing incubation time in carbachol, but the effect was most pronounced in type I fibers (Fig. 1). This finding accords with our observation that the most severe contractures occurred predominantly within type I fibers. The effects appeared to be reversible. Indeed, after rinsing away the carbachol, the ratios were in the normal range (Fig. 1). The quotient between the results of type I and type II fibers declined with increasing incubation time in carbachol, corresponding to an augmentation of the difference between these two fiber types. After rinsing away the carbachol, these differences were also restored, indicating that mostly type I fibers recover after the carbachol depletion.

\section{DISCUSSION}

This study has confirmed an earlier report ${ }^{1}$ that transient stimulation of AChRs with carbachol leads to local and reversible calcium-dependent

FIGURE 3. Overview of longitudinally sectioned MEPs with underlying contractures. (A) Beneath the MEP, the sarcomeric striations are condensed into a band-like contracture. Given the presence of numerous, subsarcolemmal mitochondria $(\mathrm{sm})$, the muscle fiber is classified as type I. SN, soleplate nucleus (10minute carbachol incubation used-protocol $\mathrm{E}_{2}$; semithin section; bar $=10 \mu \mathrm{m}$ ). (B) Overview of a motor endplate with two spot-like contractures. Owing to the plane of sectioning, the loop-like arborization of the axon terminal (AT) has been cut twice. The sarcoplasm of the MEP has been squeezed out of the muscle fiber and overlaps it on one side $(\varpi)$. Given the absence of subsarcolemmal mitochondria, this fiber is classified as type II. SN, soleplate nucleus (10-min carbachol incubation-protocol $E_{2}$; semithin section; bar $=10 \mu \mathrm{m}$ ). (C) After incubation in carbachol for 30 minutes (protocol $E_{3}$ ), a type II muscle fiber manifests a weak contracture underneath the MEP. AT, axon terminal; SN, soleplate nucleus (semithin section; bar $=10 \mu \mathrm{m}$ ). (D) Tangential section through a motor endplate of a muscle fiber, which had been exposed to carbachol for 1 minute (protocol $E_{1}$ ). The sarcoplasm of the MEP has been squeezed out of the muscle fiber and overlaps it $(\hookleftarrow)$. AT, axon terminal; $\mathrm{SN}$, soleplate nucleus (thick semithin section; bar $=10 \mu \mathrm{m}$ ). 

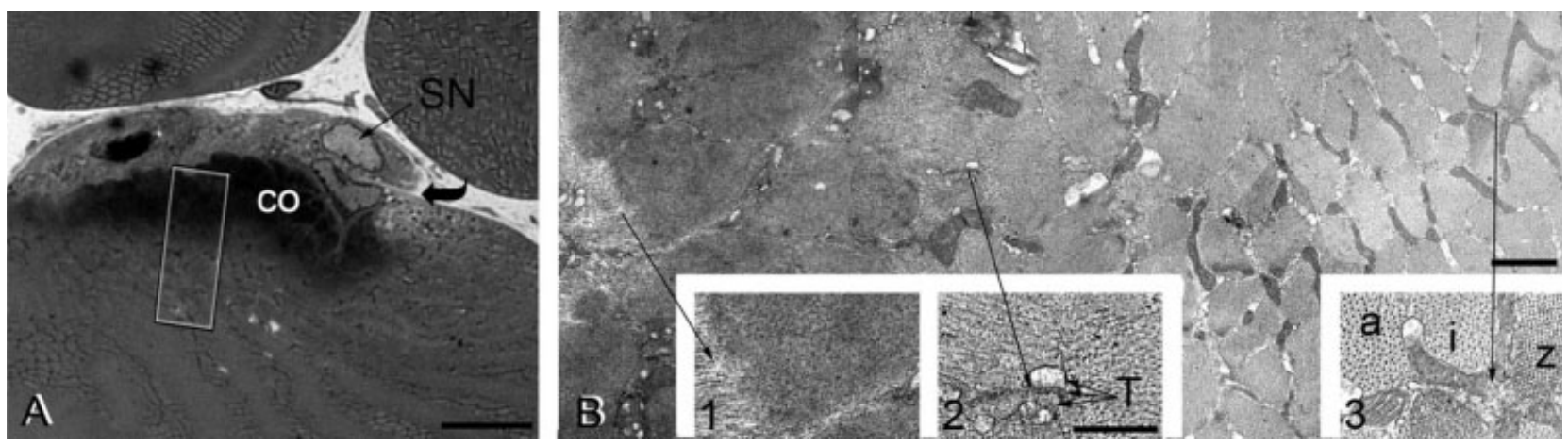

FIGURE 4. Cross-section of a muscle fiber that had been exposed to carbachol for 30 minutes (protocol $\mathrm{E}_{3}$ ). (A) The overview shows an MEP with an underlying contracture (co). Above the contracture, the sarcoplasm of the MEP has been squeezed out and overlaps the muscle fiber $(\varpi)$. SN, soleplate nucleus (semithin section; bar $=1 \mu \mathrm{m}$ ). (B) Detail of the boxed region in (A) illustrating the effect of the contracture on the structure of the A- and I-bands (ultrathin section; bar $=1 \mu \mathrm{m}$ ). Insets 1 , 2, and 3 represent higher magnification views of the indicated areas in (B). Inset 1: In this region, the punctuate pattern that is so typical of cross-sectioned myofibrils is no longer visible. Individual myosin filaments can be distinguished, but they are not arranged according to the usual hexagonal pattern. Inset 2: Deeper in the muscle fiber the register of the myofibrils is still disturbed, as evidenced by their oblique course. T, triads. Inset 3: Deep in the muscle fiber, the usual punctuate pattern of the A-band (A) and I-band (I), as well as the rectangular arrangement of actin filaments in the Z-line (Z), is visible (bar for all insets $=500 \mathrm{~nm}$.

contractures underneath MEPs. Until now, there has been no information pertaining to the spatial extent and early time-course of formation of these localized contractions, because previous studies have focused on the pathological effects on the pre- and postsynaptic sides after chronic rather than transient stimulation of AChRs. ${ }^{1,2,4,5,13}$ The present findings reveal that even a 1-minute treatment of skeletal muscle fibers with $0.1 \mathrm{mM}$ carbachol leads to fiber-type-dependent local shortening of the sarcomeres beneath the MEP, which can deform the normal beret-like shape of the MEP to a wart- or mushroom-like shape. This shortening of the sarcomeres beneath the MEP can reduce the sarcomere length to $320 \mathrm{~nm}$ or $13 \%$ of its normal value. However, these local strong shortenings are spatially confined to spots or bands beneath the MEPs and, in most cases, the sarcomeres in the up- and downstream region are stretched. Consequently, when the image containing the MEP is viewed as a whole, the combined effect of shortening and stretching leads to a contracture of $<60 \%$ of the normal resting length of a sarcomere, depending on the duration of carbachol application and the fiber type (Fig. 1). Our observations reveal that not all fibers manifest contractures to the same extent. This finding accords with the observation that the number of contractures occurring within the potassium-depolarized muscle fibers of frogs increases as a function of carbachol concentration. ${ }^{14}$ Because the carbachol concentration we used was $2 \%$ the highest that was employed, ${ }^{14}$ it is hardly surprising that not all fibers were implicated.

With respect to our own experimental set-up of carbachol application, we must consider a possible desensitization of the AChR by carbachol, ${ }^{15-17}$ which can reduce the strength of carbacholinduced contractures. Even so, short-term application of $0.1 \mathrm{mM}$ carbachol led to calcium-dependent contractures below MEPs, as it has been shown for long-term carbachol incubations, ${ }^{14,17}$ indicating that not all AChRs had yet been desensitized. The formation of scattered local shortenings along the muscle fiber outside the region of the MEP could be attributed to the activity of widely distributed extrasynaptic AChRs. ${ }^{18}$

Calcium enters the muscle fiber via opened AChRs, ${ }^{19-23}$ and this could lead to a local increase in the calcium concentration. ${ }^{19,20}$ After diffusing to the myofibrils, the calcium inward current could itself trigger sarcomere shortening. The great variability in the effectiveness to elicit contractions beneath MEPs using $\mathrm{ACh}^{19}$ indicates that the local ACh-induced increase of calcium concentration is sometimes too small to reach the necessary level for activating the shortening of sarcomeres. Assuming that a cloud-like propagation of calcium diffusion into the muscle fiber occurs in a predominantly longitudinal direction, ${ }^{24}$ and that an equalizing overlapping of individual diffusion clouds takes place from one margin of the MEP to the other, then a contracture along the entire length of the MEP and the adjacent extrajunctional sarcomeres would be expected. However, this expectation is not borne out by our own observations of spot- to band-like contractures and of stretching in adjacent sarcomeres. If the spatial location of the contractures reflects the local availability of free calcium, then the occurrence of band-like events suggests more transverse propagation of calcium diffusion as the trigger for the sarcolemmal shortening. 
However, local subsarcolemmal injections of free calcium induce highly circumscribed contractures, ${ }^{14,25}$ which accords with the circumstance that skeletal muscle fibers are not possessed of a calcium-induced calcium release. ${ }^{26}$

Our observation that the contractures are spatially confined (transversely and longitudinally) to the myofibrils that underlie MEPs suggests that the carbachol-induced MEP potential passed neither along the outer surface of the MEP to the muscle fiber nor into the T-tubules of the muscle fiber, because, otherwise, the contractures would have been more widespread and the stretching of neighboring sarcomeres would not have occurred, respectively. However, with an assumed space-constant of $20-40 \mu \mathrm{m}$ for T-tubules, ${ }^{26}$ even a low MEP potential could be propagated along those $\mathrm{T}$ tubules of the MEP that open into the subsynaptic folds $s^{27,28}$ and thereby into the deep regions of the myofibrils. Here the depolarization could induce an inward calcium current across the membrane of the T-system ${ }^{29,30}$ and calcium release through the sarcoplasmic reticulum by dihydropyridine receptor charge movements, which depend on the luminal calcium concentration. ${ }^{31}$ Such an assumption is supported by the finding that D600 (methoxyverapamil), an inactivator of dihydropyridine receptors, nearly protects muscle fibers from extracellular calcium-dependent carbacholinduced necrosis. ${ }^{1}$

Prolonged treatment with carbachol (from 30 minutes up to 3 hours) or poisoning muscles with esterase inhibitors induces contractures and an ensuing dissolution of the Z-lines beneath the MEPs. ${ }^{1,2,5,13,32}$ This calcium-dependent effect resembles the pathological changes seen in human patients with slow-channel syndrome ${ }^{3}$ and in transgenic mice with slow-closing AChRs. ${ }^{6}$ Even exercise, however, which is associated with a higher frequency of AChR activation, does not lead to a calcium overload in more than two-thirds of the fibers of these transgenic mice. ${ }^{6}$ Interestingly, in all studies that have illustrated MEPs with the underlying myofibrils after pharmacologic stimulation of the AChRs, ${ }^{1,2,13}$ these characteristic structural changes have only been observed within fibers that could be classified as type I (according to the established definition ${ }^{9,10}$ ). These observations accord with our own (Fig. 1) and with the finding that predominantly type I fibers undergo atrophy after a daily application of neostigmine. ${ }^{4}$

We must take into account the sodium channels along the fiber ${ }^{33}$ and the crest of the subsynaptic folds, ${ }^{34}$ whose function in the crest of the subsynaptic folds is to enhance the AChR-generated MEP potential. ${ }^{33,35,36}$ Slower inactivation of these sodium channels within slow muscle fibers, ${ }^{33}$ and the consequently prolonged enhancement of the MEP potential, should facilitate deeper propagation of the MEP potential into the T-system of the $\mathrm{MEP}^{27,28}$ and the adjacent T-system of the myofibrils underneath the MEP, thereby leading to stronger local contractures in slow muscle fibers.

On the other hand, we must consider that the myofibrillar proteins of slow fibers are more sensitive to calcium than fast fibers, ${ }^{37}$ which could account for the stronger contractures in slow fibers. However, the occurrence of band-like contractures and stretched sarcomeres in the neighboring areas indicates local differences in the concentration of free calcium. Although the higher calcium sensitivity of myofibrillar proteins in slow fibers will additionally augment the strength of carbachol-induced contractures, it cannot be the main reason for the differences in fiber types.

Differences in the severity of pathological changes observed in transgenic mice with manipulated AChRs may be attributed not only to differences in their levels of expression in different muscle fibers ${ }^{6}$ but also to fiber-type-specific differences in the handling of calcium.

The author thanks Professor W. Dauber (Institute of Anatomy, University of Tuebingen) and Dr. O. Baum (Institute of Anatomy, University of Bern) for helpful discussions, and to Dr. Cery England (Institute of Anatomy, University of Bern) for linguistic help.

\section{REFERENCES}

1. Leonard JP, Salpeter MM. Agonist-induced myopathy at the neuromuscular junction is mediated by calcium. J Cell Biol 1979;82. 811-819.

2. Leonard JP, Salpeter MM. Calcium-mediated myopathy at neuromuscular junctions of normal and dystrophic muscle. Exp Neurol 1982; 76:121-138.

3. Engel AG. Molecular biology of end-plate diseases. In: Salpeter MM, editor. The vertebrate neuromuscular junction. New York: Alan R. Liss; 1987. p 361-424.

4. Kawabuchi M, Osame M, Watanabe S, Igata A, Kanaseki T. Myopathic changes at the end-plate region induced by neostigmine methylsulfate. Experientia 1976;32:623-625.

5. Salpeter MM, Kasprzak H, Feng H, Fertuck H. Endplates after esterase inactivation in vivo: correlation between esterase concentration, functional response and fine structure. J Neurocytol 1979;8:95-115.

6. Gomez CM, Maselli RA, Groshong J, Zayas R, Wollmann RL, Cens T, et al. Active calcium accumulation underlies severe weakness in a panel of mice with slow-channel syndrome. J Neurosci 2002;22:6447-6457.

7. Greene EC. Anatomy of the rat. New York: Hafner; 1963.

8. Richardson KC, Jarett L, Finke EH. Embedding in epoxy resins for ultrathin sectioning in electron microscopy. Stain Technol 1960;35: 313-323.

9. Dauber W. Dicke und dünne Muskelfasern im Rectus abdominis von Ratte und Maus. Ein Vergleich licht- und elektronenmikroscopischer Befunde [Thick and thin muscle fibers in rectus abdominus of rat and mouse. Comparison of light and electron microscopical findings]. Verh Anat Ges 1973;67:657-662.

10. Dauber W. Zur Deutung des Querschnittsbildes von Skeletmuskelfasern. Licht- und Elektronenmikroskopische Untersuchungen am $\mathrm{m}$. rectus abdominis von Ratte und Maus [Evaluation of skeletal muscle cross sections. Light and electron microscopic studies of abdominal rectal muscle of rat and mouse]. Z Mikrosk Anat Forsch 1974;88: $857-870$.

11. Scheffé H. An Analysis of variance for paired comparisons. J Am Stat Assoc 1952;47:381-400.

12. Reynolds ES. The use of lead citrate at high $\mathrm{pH}$ as an electron-opaque stain in electron microscopy. J Cell Biol 1963;17:208-212.

13. Laskowski MB, Olson WH, Dettbarn WD. Initial ultrastructural abnormalities at motor end plate produced by a cholinesterase inhibitor. Exp Neurol 1977;57:13-33. 
14. Parsons RL, Nastuk WL. Activation of contractile system in depolarized skeletal muscle fibers. Am J Physiol 1969;217:364-369.

15. Edmonds B, Gibb AJ, Colquhoun D. Mechanisms of activation of muscle nicotinic acetylcholine receptors and the time course of endplate currents. Ann Rev Physiol 1995;57:469-493.

16. Katz B, Thesleff S. A study of the desensitization produced by acetylcholine at the motor end-plate. J Physiol 1957;138:63-80.

17. Manthey AA. Changes in Ca permeability of muscle-fibers during desensitization to carbamylcholine. Am J Physiol 1974;226:481-489.

18. Fambrough DM. Control of acetylcholine receptors in skeletal muscle. Physiol Rev 1979;59:165-227.

19. Allard B, Bernengo JC, Rougier O, Jacquemond V. Intracellular $\mathrm{Ca}^{2+}$ changes and $\mathrm{Ca}^{2+}$-activated $\mathrm{K}^{+}$channel activation induced by acetylcholine at the endplate of mouse skeletal muscle fibres. J Physiol 1996;494:337-349.

20. Decker ER, Dani JA. Calcium permeability of the nicotinic acetylcholine receptor: the single-channel calcium influx is significant. J Neurosci 1990;10:3413-3420.

21. Jenkinson DH, Nicholls JG. Contractures and permeability changes produced by acetylcholine in depolarized denervated muscle. J Physiol 1961;159:111-127.

22. Miledi R. Intracellular calcium and desensitization of acetylcholine receptors. Proc R Soc Lond B Biol Sci 1980;209:447-452.

23. Miledi R, Parker I, Schalow G. Transmitter induced calcium entry across the post-synaptic membrane at frog end-plates measured using arsenazo III. J Physiol 1980;300:197-212.

24. Parker I, Zang WJ, Wier WG. $\mathrm{Ca}^{2+}$ sparks involving multiple $\mathrm{Ca}^{2}$ release sites along Z-lines in rat heart cells. J Physiol 1996;497:31-38.

25. Niedergerke R. Local muscular shortening by intracellular applied calcium. J Physiol 1955;128:12P-13P.

26. Dulhunty AF, Carter G, Hinrichsen C. The membrane capacity of mammalian skeletal muscle fibres. J Muscle Res Cell Motil 1984;5: 315-332.
27. Dauber W, Voigt T, Härtel X, Meyer J. The t-tubular network and its triads in the sole plate sarcoplasm of the motor end-plate of mammals. J Muscle Res Cell Motil 2000;21:443-449.

28. Voigt T, Dauber W. About the T-system in the myofibril-free sarcoplasm of the frog muscle fiber. Tissue Cell 2004;36:245-248.

29. Almers W, Fink R, Palade PT. Calcium depletion in frog-muscle tubules-the decline of calcium current under maintained depolarization. J Physiol 1981;312:177-207.

30. Shtifman A, Paolini C, Lopez JR, Allen PD, Protasi F. $\mathrm{Ca}^{2+}$ influx through alpha(1S) DHPR may play a role in regulating $\mathrm{Ca}^{2+}$ release from RyR1 in skeletal muscle. Am J Physiol 2004;286. C73-C78.

31. Pizarro G, Fitts R, Uribe I, Rios E. The voltage sensor of excitationcontraction coupling in skeletal-muscle-ion dependence and selectivity. J Gen Physiol 1989;94:405-428.

32. Khan MA. Effects of myotoxins on skeletal muscle fibers. Prog Neurobiol 1995;46:541-560.

33. Ruff RL. Sodium channel slow inactivation and the distribution of sodium channels on skeletal muscle fibres enable the performance properties of different skeletal muscle fibre types. Acta Physiol Scand 1996;156:159-168.

34. Flucher BE, Daniels MP. Distribution of $\mathrm{Na}^{+}$channels and ankyrin in neuromuscular junctions is complementary to that of acetylcholine receptors and the $43 \mathrm{kd}$ protein. Neuron 1989;3: 163-175.

35. Martin AR. Amplification of neuromuscular-transmission by postjunctional folds. Proc R Soc Lond B Biol Sci 1994;258:321-326.

36. Wood SJ, Slater CR. The contribution of postsynaptic folds to the safety factor for neuromuscular transmission in rat fast- and slowtwitch muscles. J Physiol 1997;500:165-176.

37. Schiaffino S, Reggiani C. Molecular diversity of myofibrillar proteins: gene regulation and functional significance. Physiol Rev 1996;76: $371-423$. 